\title{
Pharmacological Antagonism of Kainate Receptor Rescues Dysfunction and Loss of Dopamine Neurons in a Mouse Model of Human Parkin-induced Toxicity
}

\section{Maria Regoni}

Universita Vita Salute San Raffaele

\section{Stefano Cattaneo}

Division of Neuroscience, IRCCS San Raffaele Scientific Institutele

\section{Daniela Mercatelli}

Universita degli Studi di Ferrara, Dipartimento di Scienze Mediche, Sezione di Farmacologia

\section{Salvatore Novello}

Universita degli Studi di Ferrara, Dipartimento di Scienze Mediche, Sezione di Farmacologia

\section{Alice Passoni}

Istituto di Ricerche Farmacologiche Mario Negri Sede di Milano

\section{Renzo Bagnati}

Istituto di Ricerche Farmacologiche Mario Negri Sede di Milano

\section{Enrico Davoli}

Istituto di Ricerche Farmacologiche Mario Negri Sede di Milano

\section{Laura Croci}

Division of Neuroscience, IRCCS San Raffaele Scientific Institute

\section{Gian Giacomo Consalez}

Universita Vita Salute San Raffaele

\section{Federica Albanese}

Universita degli Studi di Ferrara Dipartimento di Scienze MEdiche, Sezione di Farmacologia

\section{Letizia Zanetti}

Division of Nuroscience San Raffaele Scientific Institute

\section{Maria Passafaro}

Istituto di Neuroscienze Consiglio Nazionale delle Ricerche

\section{Giulia Maia Serratto}

Istituto di Neuroscienze Consiglio Nazionale delle Ricerche

\section{Alessio Di Fonzo}

Ospedale Maggiore Policlinico Neurology Unit

\section{Flavia Valtorta}

Universita Vita Salute San Raffaele

\section{Andrea Ciammola}

Istituto Auxologico Italiano Istituto di Ricovero e Cura a Carattere Scientifico

\section{Stefano Taverna}

Divisione di Neuroscienze, IRCCS San Raffaele Scientific Institute 


\section{Michele Morari}

Universita degli Studi di Ferrara

Jenny Sassone ( $\nabla$ sassone.jenny@hsr.it)

Universita Vita-Salute San Raffaele https://orcid.org/0000-0003-4854-1992

\section{Research article}

Keywords: Kainate receptor, dopaminergic neurons, parkinQ311X mouse, UBP310, excitatory synapse

Posted Date: June 24th, 2020

DOI: https://doi.org/10.21203/rs.3.rs-36149/v1

License: (a) This work is licensed under a Creative Commons Attribution 4.0 International License. Read Full License 


\section{Abstract}

\section{Background}

Mutations in the PARK2 gene encoding the protein parkin cause autosomal recessive juvenile parkinsonism (ARJP), a neurodegenerative disease characterized by dysfunction and death of dopamine (DA) neurons in the substantia nigra pars compacta. Since a neuroprotective therapy for ARJP does not exist, research efforts aimed at discovering potential targets for neuroprotection are critically needed.

A previous study demonstrated that loss of parkin function or expression of parkin mutants associated with ARJP causesan accumulation of glutamate kainate receptors (KARs) in human brain tissues and an increase of KAR-mediated currents in neuronsin vitro.

\section{Methods}

Based on the hypothesisthat such KAR hyper-activation may contribute to the death of nigralDA neurons, we investigated the effect of KAR antagonism on the DA neuron dysfunction and death that occur in the parkinQ311X mouse, a model of human parkin-induced toxicity.

\section{Results}

We found that early accumulation of KARs occurs in the DA neurons of the parkinQ311X mouse model and that chronic administration of the KAR antagonist UBP310 prevents DA neuron loss. This neuroprotective effect was associated with rescue of the abnormal firing rate of nigral DA neurons and downregulation of GluK2, the key KAR subunit.

\section{Conclusions}

This study provides novel evidence ofa causal role of glutamate KARs in the DA neuron dysfunction and loss occurring in a mouse model of human parkin-induced toxicity. Our results support KAR as a potential target in the development of a neuroprotective therapy for ARJP.

\section{Background}

Mutations in the PARK2 gene (PRKN, OMIM 600116) cause the most common form of autosomal recessive juvenile parkinsonism (ARJP), a neurodegenerative disease characterized by the loss of dopamine (DA) neurons in the substantia nigra pars compacta (SNc)[1]. The PARK2 gene encodes parkin, a ubiquitin-ligase enzyme that catalyzes the transfer of ubiquitin to protein substrates, thus regulating their trafficking and turnover [2]. Many parkin substrates have been identified to date, indicating that parkin is a multifunctional protein involved in many intracellular processes, including the control of mitochondrial integrity and the regulation of apoptosis and transcription [3,4]. These studies notwithstanding, how PARK2 mutations lead to DA neuron death is still unclear. More importantly, a therapy that prevents or slows down the neurodegeneration in ARJP patientshas not been developed yet.

Accruing evidence suggests thatparkinplays an important role in the modulation of neurotransmission [5]. Parkin localizes at both the presynaptic terminals, where it interacts with synaptic vesicles [6], and at the postsynaptic 
elements [7], where it interacts with the synaptic scaffolding molecules PSD-95, CASK (Ca ${ }^{2+} / \mathrm{CaM}^{-a s s o c i a t e d}$ serine kinase)[7] and PICK1 [8]. In a previous study, we reported that parkin interacts with and ubiquitinates the GluK2 subunits of the ionotropic glutamate kainate receptor (KAR) and that it regulates KAR currents in glutamatergic neurons [9]. Loss of parkin function was found to lead to GluK2 accumulation and increased KAR currents in glutamatergic neurons in vitro[9]. GluK2/KAR levels were increased in the brain tissues of parkinknockout mice [10] and ARJP patients bearing PARK2 mutations [9].

KARs are expressed in human DA neurons [11,12] (http://www.braineac.org), but their precise functions in DA neuron physiology have not been established to date. Since the main effect of KAR activation in glutamatergic neurons is an increase of neuronal excitability $[13,14]$, it is conceivable that activation of postsynaptic KARs expressed onSNc DA neurons increases their excitability. Therefore, KARs accumulation in DA neurons of ARJP models and patients might contribute to DA neuron dysfunction and, ultimately, death.

Here we investigated the potential role of KARs in the DA neuron dysfunction and loss that occur in the parkinQ311X mouse, a model of human parkin-induced toxicity.

\section{Methods}

\section{Animals}

C57B1/6N ParkinQ311X mice,expressing the human parkin variant parkinQ311X [15] selectively in DA neurons, were previously generated and characterized [16](The Jackson Laboratories, Bar Harbor, ME, USA). This mouse model has normal wild-type parkin alleles on both chromosomes in addition to the exogenous human parkinQ311X variant, which is ectopically expressed from a bacterial artificial chromosome and driven by the dopamine transporter (DAT) promoter. The phenotype of this mouse is due to a dominantly inherited toxic gain of function, as previously reported[16]. Gender-matched littermates were used as controls in the experiments. All experiments were conducted with the aim of minimizing the number of sacrificed animals.Mice were maintained and bred at the animal house of Ospedale San Raffaele in compliance with institutional guidelines and international laws (EU Directive 2010/63/EU EEC Council Directive 86/609, OJL 358, 1, December 12, 1987, NIH Guide for the Care and Use of Laboratory Animals, U.S. National Research Council, 1996).

\section{Dissection of substantia nigra}

The mice were anesthetized and sacrificed. The brains were removed, placed in ice-cold phosphate buffer saline

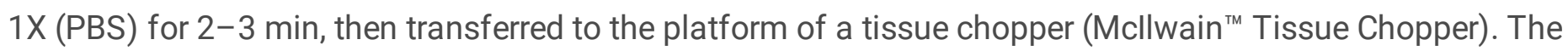
tissue was positioned perpendicular to the chopper blade and 300- $\mu \mathrm{m}$ thick coronal slices were cut in less than 1 minute. The brain slices were immediately transferred to PBS 1X. Substantia nigra was isolated under a dissecting microscope and immediately frozen in liquid nitrogen.

\section{Western blotting}

Western blotting was performed with 4-12\% NovexNuPAGE SDS-PAGE gels (Life Technologies) according to the manufacturer's protocol. Brain tissues were homogenized with lysis buffer (50 mMTris, pH 7.5, 150 mMNaCl, 1 mM EDTA, $1 \%$ NP40, protease inhibitors, $0.5 \%$ sodium deoxycholate, $0.1 \%$ SDS, complete mini protease inhibitors cocktail tablet (Roche)). The following antibodies were used: anti-GluR6/7 (Origine cod. TA310550 
1:1000), anti-GluA1 (Cell Signalling cod. 13185-D4N9V 1:2000), anti-GluA2 (NeuroMab clone L21/32 1:1000), anti-KA2 (Millipore cod. 06-315 1:2000), anti-Fodrin (Cell Signalling cod. 2122 1:1000), anti-PARP (Cell Signalling cod.9542 1:1000), anti-TH (Millipore cod. MAB310 clone LNC1 1:1000), anti-GAPDH (Santa Cruz cod. sc-25778 1:1000), anti-Tubulin $\beta 3$ (Covance cod. MMS-435P: 1:10.000).

\section{Immunohistochemistry and stereological cell count in SNc}

The micewere deeply anesthetized and transcardially perfused with $0.9 \% \mathrm{NaCl}$ solution, followed by $4 \%$ paraformaldehyde in PBS (0.1 M, pH 7.4, Sigma-Aldrich, St. Louis, MO, USA). The brains were transferred to a $30 \%$ sucrose solution in PBS for cryoprotection and stored at $-80^{\circ} \mathrm{C}$. For GluK2 labelling, the tissues were cut to30 $\mu \mathrm{m}$ thickness, transferred to glass slides coated with polylysine, and dried at room temperature. For tyrosine hydrolase $(\mathrm{TH})$ labelling, the slices were cut to $50 \mu \mathrm{m}$ thickness, rehydrated, washed, and incubated in sodium citrate buffer $(\mathrm{pH} 6)$ at $80^{\circ} \mathrm{C}$ for $30 \mathrm{~min}$, then kept for 20 min on ice. The slices were then treated with blocking solution, 5\%normal goat serum (NGS, Sigma-Aldrich) $+0.1 \%$ triton in PBS, and incubated for $48 \mathrm{~h}$ with 1:250 antiGluR6/7 (04-921, Millipore, Burlington, MA, USA) and 1:500 anti-TH (Mab318, Millipore). After three washes with PBS, the tissues were incubated for $2 \mathrm{~h}$ at room temperature with secondary antibodies (1:300 goat anti-rabbit IgG Alexa 488 to reveal GluK2 and 1:300 goat anti-mouse IgG Alexa 546 to reveal TH). Images were acquired with a Leica TCS SP2 (Leica, Wetzlar, Germany)and analysed using ImageJ software [17].

\section{Stereological cell count in SNc}

Stereological counting was performed according to published methods [18].Fifty-micrometer thick free-floating sections of SNc (AP from -3.16 to -3.52 from bregma[19]) were rinsed in PBS, incubated for 30 min at room temperature with blocking solution (PBS + BSA 1:50 + Triton X100 0.3\%), and then incubated overnight at $4^{\circ} \mathrm{C}$ with TH antibody (ab112; 1:750 in BSA 1\% PBST; Abcam, Cambridge, UK). The sections were then rinsed and incubated with a secondary antibody (ab6721, 1:500 in BSA 1\% PBST; Abcam) and revealed by a DAB substrate kit (ab64238, Abcam). The sections were mounted on gelatinized slides, dehydrated, and overlaid by coverslips. Stereological analysis was performed by counting $\mathrm{TH}+$ neurons (phenotypic marker) and cresyl violet stained cells (structural marker) in the SNc, according to an unbiased stereological sampling method based on optical fractionator stereological probe [18,20]. A Leica DM6000B motorized microscope coupled with Mercator Pro software (Mercator Digital Imaging System, Explora Nova, La Rochelle, France) was used. Counting was performed on at least 5 consecutive slices (magnified at 40X).

\section{Analyses HPLC-MRM analysis of UBP310 concentration in brain tissuesand in plasma}

Mice were injected i.p. with UBP310 (20 mg/Kg) or with an equivalent volume of vehicle. After 10-30-60-120-240480-1440 min, animals were sacrificed, plasma and brain samples were collected and stored at $-80^{\circ} \mathrm{C}$ until the analysis. Brain samples (100 mg) were homogenized in $1 \mathrm{~mL}$ of methanol/water 85:15 (v/v), containing $10 \mathrm{ng}$ of TRP-D5. Samples were then placed for $20 \mathrm{~min}$ at $-80^{\circ} \mathrm{C}$, in order to allow the separation of fat materials. Homogenates were centrifuged for $15 \mathrm{~min}$ at $13200 \mathrm{rpm}$ at $4{ }^{\circ} \mathrm{C}$ and $500 \mu \mathrm{L}$ of the supernatants were dried under nitrogen and re-suspended in $100 \mu \mathrm{L}$ of chromatographic mobile phase for instrumental analysis.

The analyses of UBP310 were performed using a 1200 Series HPLC system (Agilent Technologies, Santa Clara, CA, USA) interfaced to an API 5500 triple quadrupole mass spectrometer (Sciex, Thornhill, Ontario, Canada). The mass spectrometer was equipped with an electrospray ionization (ESI) source and was operated in positive ion 
and multiple reaction monitoring (MRM) mode to measure the product ions formed in the collision cell from the molecular ions of the analytes. In a preliminary phase, standard solutions of UBP310 and tryptophan-D5 (TRPD5), used as internal standard, were directly injected into the UBP spectrometer, to identify the best ion transitions for MRM acquisition. The identified transitions were: $\mathrm{m} / \mathrm{z} 354.1$-> 197.1 (quantification transition) and $\mathrm{m} / \mathrm{z} 354.1$-> 308.1 (qualification transition) for UBP310; m/z 210.3 -> 150.1 (quantification transition) and $\mathrm{m} / \mathrm{z} 210.3 \rightarrow 122.1$ (qualification transition) for TRP.-D5 The ion source settings were as follows: ion spray voltage, $5500 \mathrm{~V}$; curtain gas, 28; collision gas, 7; source temperature, $320^{\circ} \mathrm{C}$; ion source gas 1 and gas 2,50 and 40 psi, respectively. The HPLC separation of UBP310 and TRP-D5 was obtained with an AscentisExpress C18 column $(150 \times 2.1 \mathrm{~mm} ; 2.7 \mu \mathrm{m}$ particle size, Sigma Aldrich, St. Louis, MO), using an elution mixture composed of solvent A ( $0.05 \%$ acetic acid in water) and solvent $B$ (acetonitrile) at $30^{\circ} \mathrm{C}$. The elution gradient was from 1 to $99 \%$ of solvent B in 12 minutes; hold at $99 \%$ for 2 minutes and re-equilibration for 5 min at $10 \%$ of solvent $B$. The injection volume was $5 \mu \mathrm{l}$ and the flow rate was $180 \mu \mathrm{l} / \mathrm{min}$.

\section{Chronic treatment with UBP310 or vehicle}

UBP310 (3-(\{3-[(2S)-2-Amino-2-carboxyethyl]-5-methyl-2,6-dioxo-3,6-dihydro-1(2H)-pyrimidinyl\}methyl)-2thiophenecarboxylic acid) was purchased from ABCAM (Cambridge, UK), dissolved in 100\% DMSO at a concentration of $10 \mathrm{mg} / \mathrm{mL}$ and then diluted in $90 \%$ saline $+10 \%$ DMSO at $1 \mathrm{mg} / \mathrm{mL}$. The mice were injected i.p. with UBP310 $(20 \mathrm{mg} / \mathrm{kg})$ or an equivalent volume of vehicle ( $90 \%$ saline $+10 \%$ DMSO) every day at the same hour (8-10 am) for the duration of the treatment.

\section{Cell-attached and whole-cell patch clamp recordings of DA neurons of the SNc in mouse brain slices.}

Twenty-five-day-old mice were anesthetized by intraperitoneal injection of a mixture of ketamine/xylazine (100 $\mathrm{mg} / \mathrm{kg}$ and $10 \mathrm{mg} / \mathrm{kg}$, respectively; Sigma-Aldrich) and perfused transcardially with ice-cold artificial cerebrospinal fluid (ACSF)containing (in mM): $125 \mathrm{NaCl}, 3.5 \mathrm{KCl}, 1.25 \mathrm{NaH}_{2} \mathrm{PO}_{4}, 2 \mathrm{CaCl}_{2}, 25 \mathrm{NaHCO}_{3}, 1 \mathrm{MgCl}_{2}$, and $11 \mathrm{D}$-glucose, saturated with $95 \% \mathrm{O}_{2}$ and $5 \% \mathrm{CO}_{2}(\mathrm{pH} 7.3)$. After decapitation, the brains were removed and $300 \mu \mathrm{m}$-thick coronal slices containing the SN were cut in ACSF at $4^{\circ} \mathrm{C}$ on a VT1000S vibratome (Leica Microsystems, Wetzlar, Germany). Individual slices were submerged in a recording chamber mounted on the stage of an upright BX51WI microscope (Olympus, Japan) equipped with differential interference contrast optics (DIC). The slices were continuously perfused with ACSF at a rate of $2-3 \mathrm{ml} / \mathrm{min}$ at $32^{\circ} \mathrm{C}$. DA neurons were recorded using glass pipettes filled with a solution containing the following (in $\mathrm{mM}$ ): $10 \mathrm{NaCl}, 124 \mathrm{KH}_{2} \mathrm{PO}_{4}, 10$

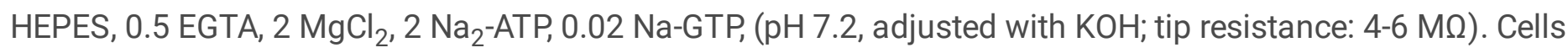
were recordedin cell-attached configurationfor spontaneous firing frequencies and whole-cell configuration for intrinsic membrane parameters. All recordings were performed using a MultiClamp 700B amplifier interfaced with a computer through a Digidata 1440A (Molecular Devices, Sunnyvale, CA, USA). Traces were sampled at a frequency of $10 \mathrm{kHz}$ and low-pass filtered at $2 \mathrm{kHz}$. Data were acquired using pClamp10 software (Molecular Devices) and analyzed with Clampfit and GraphPad Prism (La Jolla, CA).

\section{Data presentation and statistical analysis}

Data are presented as mean \pm standard error of the mean (SEM). The normality test (Kolmogorov-Smirnov test) and equal variance test (Bartlett's test)were applied. Two-tailed unpaired Student's t-test was used to compare two groups of data. One-way ANOVA followed by appropriate post hoc tests was used to compare more than two 
groups. The Friedman repeated-measure ANOVA on rankswas usedon non-normally distributed datasets to analyze the effect of UBP310 on firing frequency.

\section{Results}

\section{ParkinQ311X mice display early KAR accumulation associated with degeneration of SNc DA neurons}

We previously found that PARK2 mutations induce GluK2/KAR accumulation in ratneurons grownin vitro and in human brains [9]. To test whether PARK2 mutations induce early accumulation of KAR specifically in nigral DA neurons, we analysed GluK2 levels in the isolated SNc of 1-month-old parkinQ311X mice and WT littermates (Supplementary Fig. S1a-b). Nigral GluK2 levels were higher in the parkinQ311X mice than in WT mice. The GluA1 subunit levels of AMPAR wereunchanged (Fig. 1a). Immunofluorescence for GluK2 and TH in mesencephalic brain slices confirmed the GluK2 accumulation in the DA neurons of the parkinQ311X mice (Fig. 1b).

A 40\% reduction of the number of DA neurons in the SNc of parkinQ311X mice at 16 months of age was previously reported [16]. We analysed the number of nigral DA neurons inmice at younger ages. No differenceswere found between the parkinQ311X mice and their littermate controls at 1 month, and a $20 \%$ reduction in DA neurons was found at 6 months (Fig. 1c). These results confirm that the parkinQ311X mouse displays DA neuron loss[16] and show that degeneration of DA neurons is not limited to aged animals. Importantly, GluK2/KAR accumulation precedes DA neuron degenerationin this model of human parkin-induced toxicity.

\section{UBP310 rescues the loss of SNc DA neurons in ParkinQ311X mice}

We hypothesized that if GluK2/KAR accumulation contributes to DA neuron loss in parkinQ311X mice, then pharmacological antagonism of KAR might provide neuroprotection. UBP310 is a competitive orthosteric antagonist of KARs shown to antagonize KAR activity in vitro and in vivo[21,22]. Several lines of evidence show that UBP310 is a specific KAR antagonist[21-23].

To quantify the bioavailability and distribution of peripherally administered UBP310 in the brain, WT mice were treated with a single intraperitoneal injection of UBP310 (20mg/ $\mathrm{kg}$ i.p.) and a time-course analysis of UBP310 levels in the plasma and brain was performed. The UBP310 plasmatic peak occurred within 10 minfrominjection; UBP310 was quickly distributed in the brain, its concentration remained $>100 \mathrm{ng}$ UBP310/g brain tissue for $1 \mathrm{~h}$ before slowly diminishing within $24 \mathrm{~h}$ (Supplementary Fig. S2). No abnormal behaviours or clinical signs were observed in the treated mice: all remained presumptively healthy without signs of debilitation, pain or discomfort. Treatment did not change the levels of proteins associated with cell death (Supplementary Fig. S3a) or of neuronal and dopaminergic markers (Supplementary Fig. S3b), thus ruling out overt, acute UBP310 in vivotoxicity.

Based on these results, we treated 3-month-old parkinQ311X mice and their age-matched WT littermates with UBP310 (20mg/kg daily i.p.) or vehicle (10\% DMSO in saline) for 90 days. Chronic treatment with either UBP310 or vehicle induced no mortality or weight changes (Supplementary Fig. S4). No signs of pain, distress or toxicity 
were observed; animals fed, walked, and groomed normally. At the end of treatment, 6-month-old mice were sacrificed and brains analysed for the number of SNc DA neurons. A $~ 20 \%$ reduction in DA neurons was observed in the parkinQ311X mice treated with vehicle, whereas no significant loss of DA neuronswas found in the UBP310-treated parkinQ311X mice. To substantiate these data, a cohort of parkinQ311X mice was treated with UBP310 (20mg/kg/day i.p) for 135 days, starting from 1.5 months of age. Again, no nigral DA neuron loss was observed,demonstratingthat UBP310 is able to prevent SNc degeneration in parkinQ311X mice(Fig. 2).

\section{UBP310 normalizes firing activity of DA neurons and downregulates GluK2 levels in ParkinQ311X mice}

We investigated the mechanism by which UBP310 rescues DA neuron loss in parkinQ311X mice. The main effect of the glutamate-induced activation of KARs in hippocampal neurons is to increase neuronal excitability $[13,14]$. Conceivably,an increase in GluK2/KAR function at the glutamatergic post-synapse of SNc DA neurons might enhance DA neuron excitability, increase energy demand, and possibly result in cell death[24]. To test this hypothesis, we analysed the electrophysiological properties of SNc DA neurons in the brain slices from parkinQ311X and WT mice. Intrinsic membrane properties did not differ between WT and parkinQ311X DA neurons (Supplementary Fig. S5). However, the average spontaneous firing frequency was greater in parkinQ311X DA neurons than WT DA neurons (Fig. 3a-b). To test whether this dysfunction was mediated by KAR currents, we recorded spontaneous firing activity upon acute UBP310 application to brain slices. In WT DA neurons, $5 \mu \mathrm{M}$ UBP310 was ineffective, whereas $10 \mu \mathrm{M}$ UBP310 slightly decreased the firing frequency.In parkinQ311X DA neurons, both $5 \mu \mathrm{M}$ and10 $\mu \mathrm{M}$ UBP310 decreased the abnormal firing frequency to a level similar to WT control (Fig. 3c). These data demonstrate that pharmacological antagonism of KAR normalizes the abnormal firing frequency of PARK2 neurons.

Since pharmacological antagonism can induce receptor downregulation, we also tested whether KAR blockade by UBP310 was associated with GluK2/KAR downregulation. To do this, we analysed GluK2 levels in whole brain lysates of WT and parkinQ311X mice acutely treated with $20 \mathrm{mg} / \mathrm{kg}$ UBP310 i.p., at 4 or $8 \mathrm{~h}$ after treatment. UBP310 induced a time-dependent decrease in GluK2 levels in both WT and parkinQ311X mice: WT mice showed a significant decrease in GluK2 protein levels 4 and $8 \mathrm{~h}$ after treatment with UBP310, parkinQ311X mice showed a significant decrease in GluK2 protein levels8 $\mathrm{h}$ after treatment with UBP310 (Fig. 4a). This effect was specific for KAR,since UBP310 did not affect the levels of the AMPAR subunits GluA1 and GluA2. To confirm this result in DA neurons, we analysed GluK2 levels in mesencephalic brain slices by immunofluorescence. The experiments confirmed that UBP310 reduces GluK2 levels in the DA neurons of WT and parkinQ311X mice (Fig. 4b). The data suggest that, by binding to KAR and blocking its opening, UBP310 can also induce degradation of the key subunit GluK2.

\section{Discussion}

With this study we provide novel evidence of the role of glutamate receptor KARs in the DA neuron dysfunction and loss occurring in the parkinQ311X mouse, a model of human parkin-induced toxicity.

The potential target investigated in this study is KAR, a tetrameric receptor composed of various combinations of five subunits (named GluK1-5) expressed in many human brain regions, including SNc[12] (http://www.braineac.org/). GluK2, that promotes the cell surface expression of the other subunits [25], is the 
most abundant subunit in $\mathrm{SNc}$ [12]. Our result showing increased levels of GluK2/KARs in DA neuronsofparkinQ311X mice is in agreement with previous studies demonstrating KAR accumulation in ARJP models and in human brain tissues from ARJP patients $[9,10]$. Since wild-type parkin interacts with the C-terminal tail of GluK2 and is able to ubiquitinate GluK2 [9], GluK2 accumulation in parkinQ311X-DA neurons may result from decreased proteasome-mediated degradation. We speculate that GluK2 ubiquitination by parkin might also modulate KAR endo-exocytosis, surface localization or lateral diffusion between the synaptic and the extrasynaptic membrane of KARs.

The function of KARs in DA neurons is still unexplored. Spontaneous firing frequency sensitive to UBP310 is increasedin parkinQ311X-DA neurons. These data suggest that KARs can participate in the regulation of DA neuron firing frequency. The spontaneous activity pattern of adult SNc DA neurons in vivo results from interactions between intrinsic pacemaker mechanisms and synaptic inputs. According to the literature, however, only the pacemaker-like pattern can be recorded in vitro[26-28]. Our in vitro data suggest that the expression of parkin mutants in SNc DA neurons affects their pacemaker activity. Which might be the mechanism by which KAR receptor regulates spontaneous firing in DA neurons? Pacemaker activity is associated with DA release and intrinsically driven by voltage-gated Cav1.3 calcium channels. We speculate that KAR channel gating may facilitate membrane depolarization and activation of voltage-gated Cav1.3 calcium channels. Moreover, since KARs can activate $G$ protein signaling by stimulating phospholipase $C$ and PKC, we speculate that KAR activation can induce post-translational modifications inthe proteins involved in firing control. A previous study showed an increase in the spontaneous firing frequency of DA neurons in a PD model generated by overexpressing mutant a-synuclein[29]. This suggests that the increase in the spontaneous firing of DA neurons may be a common feature of PD forms and that UBP310 may revert this dysfunction.

Because of their large fractional $\mathrm{Ca}^{2+}$ influx during action potential and their enormously large axonal tree, SNc DA neurons have a constitutive higher metabolic demand than other neurons [30]. By increasing KAR-mediated glutamatergic transmission, PARK2 mutations can lead to early dysregulation of pacemaker activity and $\mathrm{Ca}^{2+}$ influx in SNc DA neurons, thus increasing their energy costs and susceptibility to oxidative damage, $\mathrm{Ca}^{2+}$ overload, and excitotoxicity. Consistent with this hypothesis, we found that treatment with KAR antagonist UBP310 for 3 and 4.5 months in vivo prevents DA neuron degeneration caused by parkinQ311X expression.

This finding is in line with the results of a recent study that showed that UBP310 can rescue DA neuron loss induced by the parkinsonian toxin 1-methyl-4-phenyl-1,2,3,6-tetrahydropyridine (MPTP) in mice [31], further confirming the disease-modifying potential of this approach in PD.

The molecular genetics of the parkinQ311X model differs from ARJP in human. However, overexpression of human mutant parkin is a strategy extensively used in in vitro experiments [32,33] and also in Drosophila[34,35]. Hence, in agreement with other authors, transgenic models overexpressing mutant human parkin might represent an attractive alternative to parkin deficiency models in target identification and validation studies [36].

UBP310 has been reported to be a potent antagonist at KARs [21-23,37,38].Studies in dorsal root C fibers, motoneurons and recombinant AMPAR and KAR subunits showed that UBP310 is 4000-fold more potent antagonist at KAR than AMPAR [23], and that UBP310 is ineffective at NMDAR and metabotropic glutamate receptors [23]. UBP310 blocked postsynaptic KAR at hippocampal mossy fiber CA3 synapses while sparing AMPAR and NMDAR [21]. The specificity for KAR was further demonstrated by a study showing that UBP310 is 
ineffective in preventing epileptic activity in mice knock-out for GluK2 subunit of KAR [22]. Hence, several lines of evidence show that UBP310is a specific KAR antagonist.

We found that KAR antagonism in parkinQ311X mice reduced the GluK2 levels in brain tissues and in DA neurons; this finding confirms the effects of UBP310 on KARs.In WT mice, UBP310 decreased GluK2 levels in 4h, whereas in parkinQ311X mice the decrease inGluK2 levels occurred 8h after the treatment. Considering that parkin ubiquitinates GluK2 and this post-transductional modification may mediate GluK2 turnover, we can hypothesize that mutant parkin expression may slow down GluK2 turnover. As previously suggested, it is still possible that the neuroprotective effect of UBP310 in the midbrain may result from its action on KARs formed by the combination of different subunits [31].

\section{Conclusions}

This study provides novel evidence of a causal role of glutamate KARs in the DA neuron dysfunction and loss occurring in a mouse model of human parkin-induced toxicity. Our results strongly support KAR as a novel target in neuroprotective therapy for nigral DA neurons. The favorable safety profile of chronic UBP310 in mice, along with the lack of signs of toxicity in nigral DA neurons, may facilitate this approach and pave the way to further analyses, which might extend the favourable effect of KAR antagonists to other preclinical models of human ARJP as well as of idiopathic PD.

\section{List Of Abbreviations}

ARJP:Autosomal Recessive Juvenile Parkinsonism

DA:Dopamine

KARs:Kainate receptors

DAT: Dopamine Transporter

SNc: Substantia Nigra Pars Compacta

HPLC-MRM: High Performance Liquid Chromatography-Multiple Reaction Monitoring

AMPAR:a-amino-3-hydroxy-5-methyl-4-isoxazolepropionic Acid Receptor

NMDAR: N-Methyl-d-aspartic Acid Receptor

\section{Declarations}

\section{Ethics approval}

Mice were maintained and bred at the animal house of Ospedale San Raffaele in compliance with institutional guidelines and international laws (EU Directive 2010/63/EU EEC Council Directive 86/609, OJL 358, 1, December 12, 1987, NIH Guide for the Care and Use of Laboratory Animals, U.S. National Research Council, 1996). All experiments were conducted with the aim of minimizing the number of sacrificed animals. 


\section{Consent for publication}

Not applicable.

\section{Availability of data and materials}

Not applicable

\section{Competing interests}

The authors declare that they have no competing interests.

\section{Funding}

This project was supported by the Michael J. Fox Foundation for Parkinson's Research (Grant MJFF14110 to JS), Italian Ministry of Health (GR-2016-02361366 to JS), and the Telethon Foundation (Grant GGP15167 to AC).

\section{Authors' contributions}

Conception and design of the study AC and JS. Acquisition of data MR, SC, DM, SN, AP, RB, ED, LC, FA, LZ, GMS, and ST. Analysis of data MR, SC, ST, MM, and JS. Drafting the article AC, MM and JS. Revision for important intellectual content RB, ED, GGC, MP, ADF, FV, AC, ST, and MM. All authors read and approved the final manuscript.

\section{References}

1. Kitada T, Asakawa S, Hattori N, Matsumine H, Yamamura Y, Minoshima S, et al. Mutations in the parkin gene cause autosomal recessive juvenile parkinsonism. Nature. 1998;392:605-8.

2. Zhang C-W, Hang L, Yao T-P, Lim K-L. Parkin Regulation and Neurodegenerative Disorders. Front Aging Neurosci. 2015;7.

3. Scarffe L a., Stevens D a., Dawson VL, Dawson TM. Parkin and PINK1: Much more than mitophagy. Trends Neurosci. Elsevier Ltd; 2014;37:315-24.

4. Charan RA, LaVoie MJ. Pathologic and therapeutic implications for the cell biology of parkin. Mol Cell Neurosci. 2015;66:62-71.

5. Sassone J, Serratto G, Valtorta F, Silani V, Passafaro M, Ciammola A. The synaptic function of parkin. Brain. 2017;140.

6. Kubo S, Kitami T, Noda S, Shimura H, Uchiyama Y, Asakawa S, et al. Parkin is associated with cellular vesicles. 2001;42-54.

7. Fallon L, Moreau F, Croft BG, Labib N, Gu W, Fon EA. Parkin and CASK / LIN-2 Associate via a PDZ-mediated Interaction and Are Co-localized in Lipid Rafts and Postsynaptic Densities in Brain *. 2002;277:486-91.

8. Joch M, Ase AR, Chen CX, Macdonald PA, Kontogiannea M, Corera AT, et al. Parkin-mediated Monoubiquitination of the PDZ Protein PICK1 Regulates the Activity of Acid-sensing lon Channels. 2007;18:3105-18.

9. Maraschi A, Ciammola A, Folci A, Sassone F, Ronzitti G, Cappelletti G, et al. Parkin regulates kainate receptors by interacting with the GluK2 subunit. Nat Commun. 2014;5:5182. 
10. Cremer JN, Amunts K, Schleicher A, Palomero-Gallagher N, Piel M, Rösch F, et al. Changes in the expression of neurotransmitter receptors in Parkin and DJ-1 knockout mice - A quantitative multireceptor study. Neuroscience. 2015;311:539-51.

11. Porter RHP, Eastwood SL, Harrison PJ. Distribution of kainate receptor subunit mRNAs in human hippocampus, neocortex and cerebellum, and bilateral reduction of hippocampal GluR6 and KA2 transcripts in schizophrenia. Brain Res. 1997;

12. Mueller HT, Haroutunian V, Davis KL, Meador-Woodruff JH. Expression of the ionotropic glutamate receptor subunits and NMDA receptor-associated intracellular proteins in the substantia nigra in schizophrenia. Mol Brain Res. 2004;

13. Lerma J, Marques JM. Kainate receptors in health and disease. Neuron. 2013.

14. Contractor A, Mulle C, Swanson GT. Kainate receptors coming of age: Milestones of two decades of research. Trends Neurosci. 2011.

15. Hattori N, Matsumine H, Asakawa S, Kitada T, Yoshino H, Elibol B, et al. Point mutations (Thr240Arg and Ala311Stop) in the Parkin gene. Biochem Biophys Res Commun. 1998;

16. Lu X-H, Fleming SM, Meurers B, Ackerson LC, Mortazavi F, Lo V, et al. Bacterial artificial chromosome transgenic mice expressing a truncated mutant parkin exhibit age-dependent hypokinetic motor deficits, dopaminergic neuron degeneration, and accumulation of proteinase K-resistant alpha-synuclein. J Neurosci [Internet]. 2009;29:1962-76. Available from:

http://www.ncbi.nlm.nih.gov/pubmed/19228951\%5Cnhttp://www.pubmedcentral.nih.gov/articlerender.fcgi? artid=PMC2803056

17. Schneider CA, Rasband WS, Eliceiri KW. NIH Image to ImageJ: 25 years of image analysis. Nat. Methods. 2012.

18. Novello S, Arcuri L, Dovero S, Dutheil N, Shimshek DR, Bezard E, et al. G2019S LRRK2 mutation facilitates asynuclein neuropathology in aged mice. Neurobiol Dis. 2018;

19. Paxinos G, Franklin KBJ. Paxinos and Franklin's the Mouse Brain in Stereotaxic Coordinates. Acad. Press. 2001.

20. Arcuri L, Viaro R, Bido S, Longo F, Calcagno M, Fernagut PO, et al. Genetic and pharmacological evidence that endogenous nociceptin/orphanin FQ contributes to dopamine cell loss in Parkinson's disease. Neurobiol Dis. 2016;89:55-64.

21. Pinheiro PS, Lanore F, Veran J, Artinian J, Blanchet C, Crépel V, et al. Selective block of postsynaptic kainate receptors reveals their function at hippocampal mossy fiber synapses. Cereb Cortex. 2013;

22. Peret A, Christie LA, Ouedraogo DW, Gorlewicz A, Epsztein JÔ, Mulle C, et al. Contribution of Aberrant GluK2Containing Kainate Receptors to Chronic Seizures in Temporal Lobe Epilepsy. Cell Rep. 2014;

23. Dolman NP, More JCA, Alt A, Knauss JL, Pentikäinen OT, Glasser CR, et al. Synthesis and pharmacological characterization of N3-substituted willardiine derivatives: Role of the substituent at the 5-position of the uracil ring in the development of highly potent and selective GLUK5 kainate receptor antagonists. J Med Chem. 2007;

24. Fricker M, Tolkovsky AM, Borutaite V, Coleman M, Brown GC. Neuronal cell death. Physiol. Rev. 2018.

25. Jaskolski F, Coussen F, Mulle C. Subcellular localization and trafficking of kainate receptors. Trends Pharmacol. Sci. 2005. 
26. Richards CD, Shiroyama T, Kitai ST. Electrophysiological and immunocytochemical characterization of GABA and dopamine neurons in the substantia nigra of the rat. Neuroscience. 1997;

27. Morikawa H, Paladini CA. Dynamic regulation of midbrain dopamine neuron activity: Intrinsic, synaptic, and plasticity mechanisms. Neuroscience. 2011.

28. Roeper J. Dissecting the diversity of midbrain dopamine neurons. Trends Neurosci. 2013.

29. Subramaniam M, Althof D, Gispert S, Schwenk J, Auburger G, Kulik A, et al. Mutant -Synuclein Enhances Firing Frequencies in Dopamine Substantia Nigra Neurons by Oxidative Impairment of A-Type Potassium Channels. J Neurosci. 2014;

30. Bolam JP, Pissadaki EK. Living on the edge with too many mouths to feed: Why dopamine neurons die. Mov Disord. 2012;

31. Stayte S, Laloli KJ, Rentsch P, Lowth A, Li KM, Pickford R, et al. The kainate receptor antagonist UBP310 but not single deletion of GluK1, GluK2, or GluK3 subunits, inhibits MPTP-induced degeneration in the mouse midbrain. Exp Neurol. 2020;

32. Fiesel FC, Caulfield TR, Moussaud-Lamodière EL, Ogaki K, Dourado DFAR, Flores SC, et al. Structural and Functional Impact of Parkinson Disease-Associated Mutations in the E3 Ubiquitin Ligase Parkin. Hum Mutat. 2015;

33. Yi W, Macdougall EJ, Tang MY, Krahn Al, Gan-Or Z, Trempe JF, et al. The landscape of Parkin variants reveals pathogenic mechanisms and therapeutic targets in Parkinson's disease. Hum Mol Genet. 2019;

34. Sang TK, Chang HY, Lawless GM, Ratnaparkhi A, Mee L, Ackerson LC, et al. A Drosophila model of mutant human parkin-induced toxicity demonstrates selective loss of dopaminergic neurons and dependence on cellular dopamine. J Neurosci. 2007;

35. Wang C, Lu R, Ouyang X, Ho MWL, Chia W, Yu F, et al. Drosophila overexpressing parkin R275W mutant exhibits dopaminergic neuron degeneration and mitochondrial abnormalities. J Neurosci. 2007;

36. Dawson TM, Ko HS, Dawson VL. Genetic Animal Models of Parkinson's Disease. Neuron. 2010.

37. Atlason PT, Scholefield CL, Eaves RJ, Mayo-Martin MB, Jane DE, Molnár E. Mapping the ligand binding sites of kainate receptors: Molecular determinants of subunit-selective binding of the antagonist [ $3 \mathrm{H}] \mathrm{UBP} 310$. Mol Pharmacol. 2010;

38. Perrais D, Pinheiro PS, Jane DE, Mulle C. Antagonism of recombinant and native GluK3-containing kainate receptors. Neuropharmacology. 2009;

\section{Figures}


Figure 1

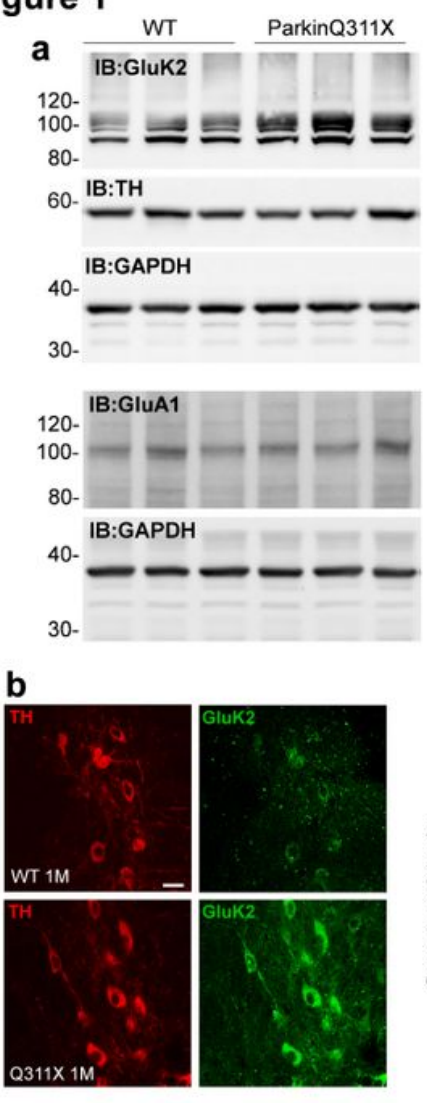

C
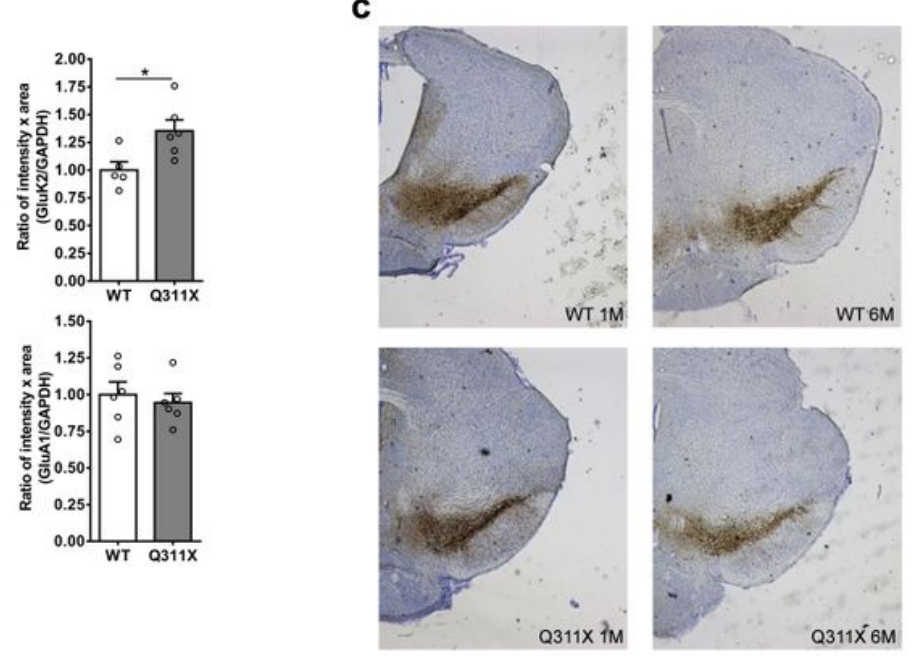

Q311X́6m
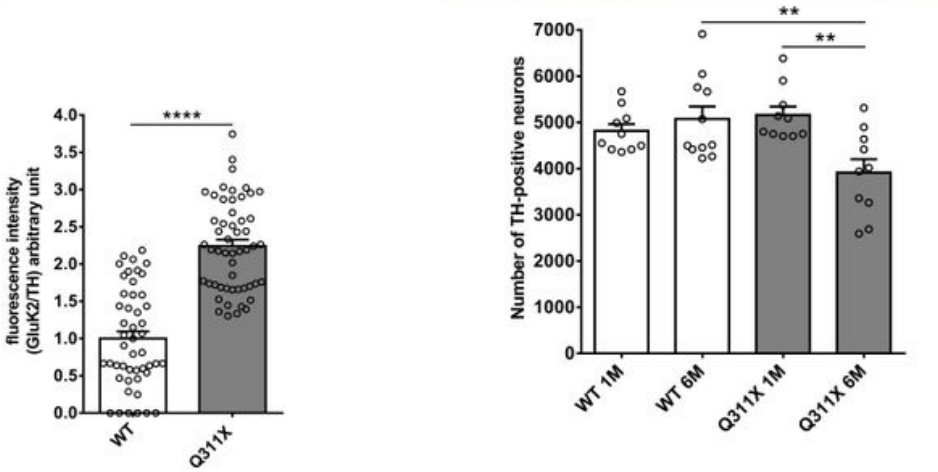

\section{Figure 1}

SNc DA neurons of parkinQ311X mice display early KAR accumulation and degeneration. a) Representative Western blot showing the levels of GluK2 subunit of KAR and GluA1 subunit of AMPAR in lysates prepared from the substantia nigra of WT or parkinQ311X mice at 1 month of age. Membranes were also probed for TH as DA neuron marker. The histograms on the right show the means \pm SEM from densitometer quantifications. GluK2: WT $1.00 \pm 0.07 \mathrm{n}=5$ mice, parkinQ311X 1.35 $\pm 0.10 \mathrm{n}=6$ mice, two-tailed unpaired Student $\mathrm{t}$ test * $\mathrm{p}=0.012 \mathrm{t}=2.786 \mathrm{df}=9$; GluA1: WT 1.00 $\pm 0.09 n=6$ mice, parkinQ311X 0.94 $\pm 0.06 n=6$ mice, two-tailed unpaired Student $t$ test $p>0.05)$. b) Representative confocal images showing TH (red) and GluK2 (green) double immunofluorescence in the SNc of the WT and the parkinQ311X mice at 1 month of age. The image is representative of 12 sections derived from $\mathrm{n}=3$ WT mice and 12 sections from $\mathrm{n}=3$ parkinQ311X mice (GluK2 antibody Millipore 04-921). Bar is 40 $\mu \mathrm{m}$. The histogram showsthe means \pm SEM of fluorescence intensity. GluK2/TH: WT 1.00 $\pm 0.10 \mathrm{n}=48$ neurons, parkinQ311X 2.24 $\pm 0.08 n=53$ neurons, two-tailed unpaired Student $t$ test $* \star \star \star p=3.87 E-16 t=9.749 \mathrm{df}=99$. Data from non normalized GluK2 intensity: WT 1.00 $\pm 0.12 \mathrm{n}=48$ neurons, parkinQ311X 3.22 $\pm 0.13 \mathrm{n}=53$ neurons, twotailed unpaired Student $t$ test $* \star \star \star p=5.03 E-22 t=12.47 \mathrm{df}=99$. c) Representative images showing $\mathrm{TH}$ immunoperoxidase labelling in the SNc of the WT and the parkinQ311X mice at 1 and 6 months of age. The histograms just below the picture show DA neuron quantification performed by stereological count. Data are the means \pm SEM of TH positive neurons: WT 1 month (1M) 4817 $\pm 146 n=10$ mice, parkinQ311X 1M 5160 $\pm 182 n=10$ mice, WT 6 months (6M) 5075 \pm 270 n=11 mice, parkinQ311X 6M 3913 \pm 293 n=10 mice, one-way ANOVA followed by Tukey's test. F=5.933, WT 6M vs. parkinQ311X 6M **p=0.0054, parkinQ311X 1M vs. parkinQ311X $6 \mathrm{M} * * \mathrm{p}=0.0033$. 


\section{Figure 2}
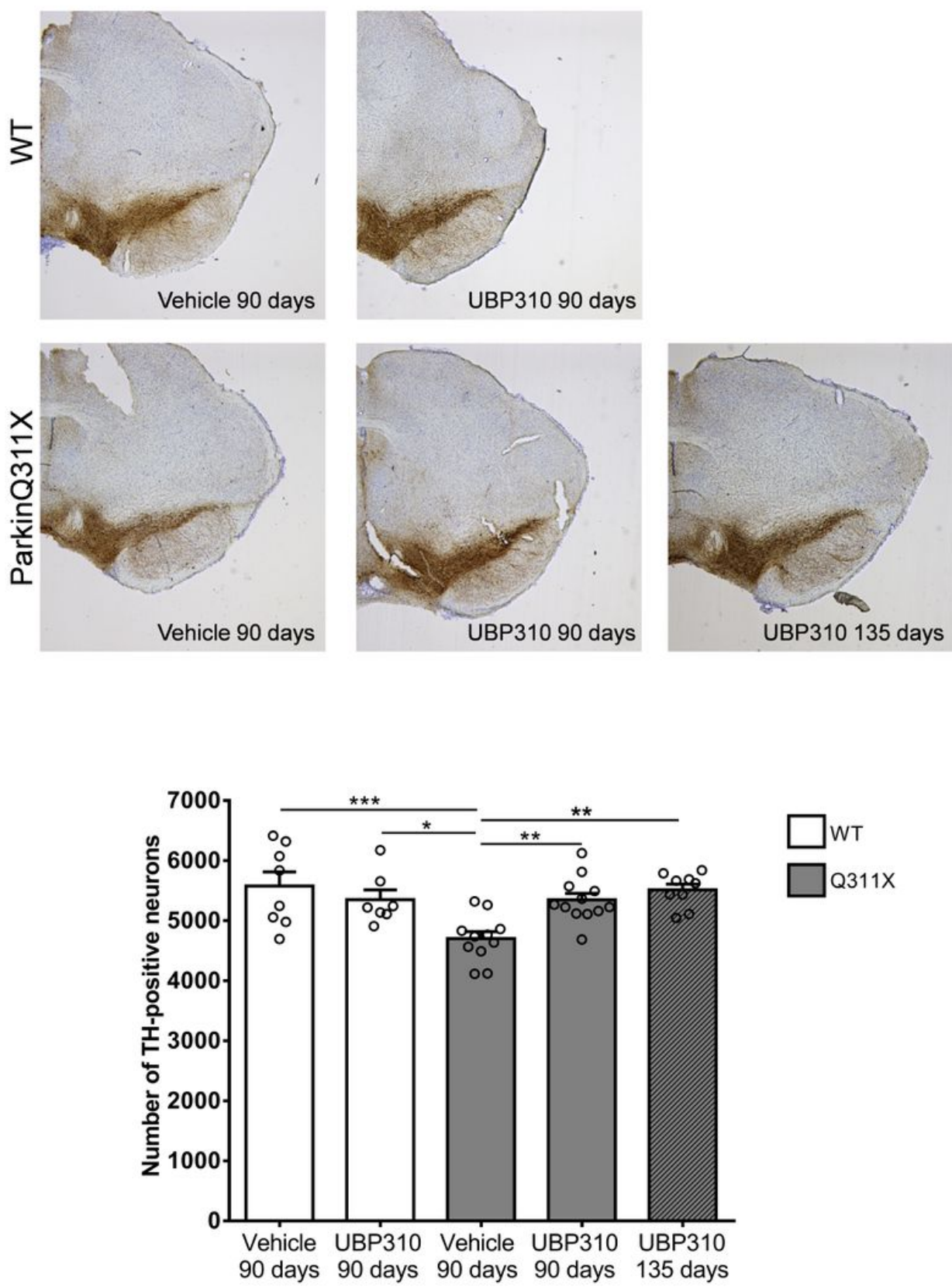

\section{Figure 2}

UBP310 rescues the loss of SNc DA neurons in parkinQ311X mice. Representative images showing TH immunoperoxidase labelling in the SNc of the WT and the parkinQ311X mice (6 months of age) treated with either vehicle or UBP310 ( $20 \mathrm{mg} / \mathrm{kg}$ i.p.) for 90 or 135 days. The graph below the picture shows DA neuron quantification performed by stereological count. Data are the means \pm SEM of TH positive neurons: WT treated with vehicle for 90 days $5578 \pm 235 n=8$ mice, WT treated with UBP310 for 90 days $5351 \pm 162 n=7$ mice, parkinQ311X treated with vehicle for 90 days $4701 \pm 117 n=11$ mice, parkinQ311X treated with UBP310 for 90 days $5347 \pm 107 n=12$ mice, parkinQ311X mice treated with UBP310 for 135 days $5514 \pm 94 n=9$ mice, one-way ANOVA followed by Tukey's test $F=6.681$, WT vehicle 90 days vs. parkinQ311X vehicle 90 days ${ }^{\star \star *} p=0.0007$, WT 


\section{Figure 3}
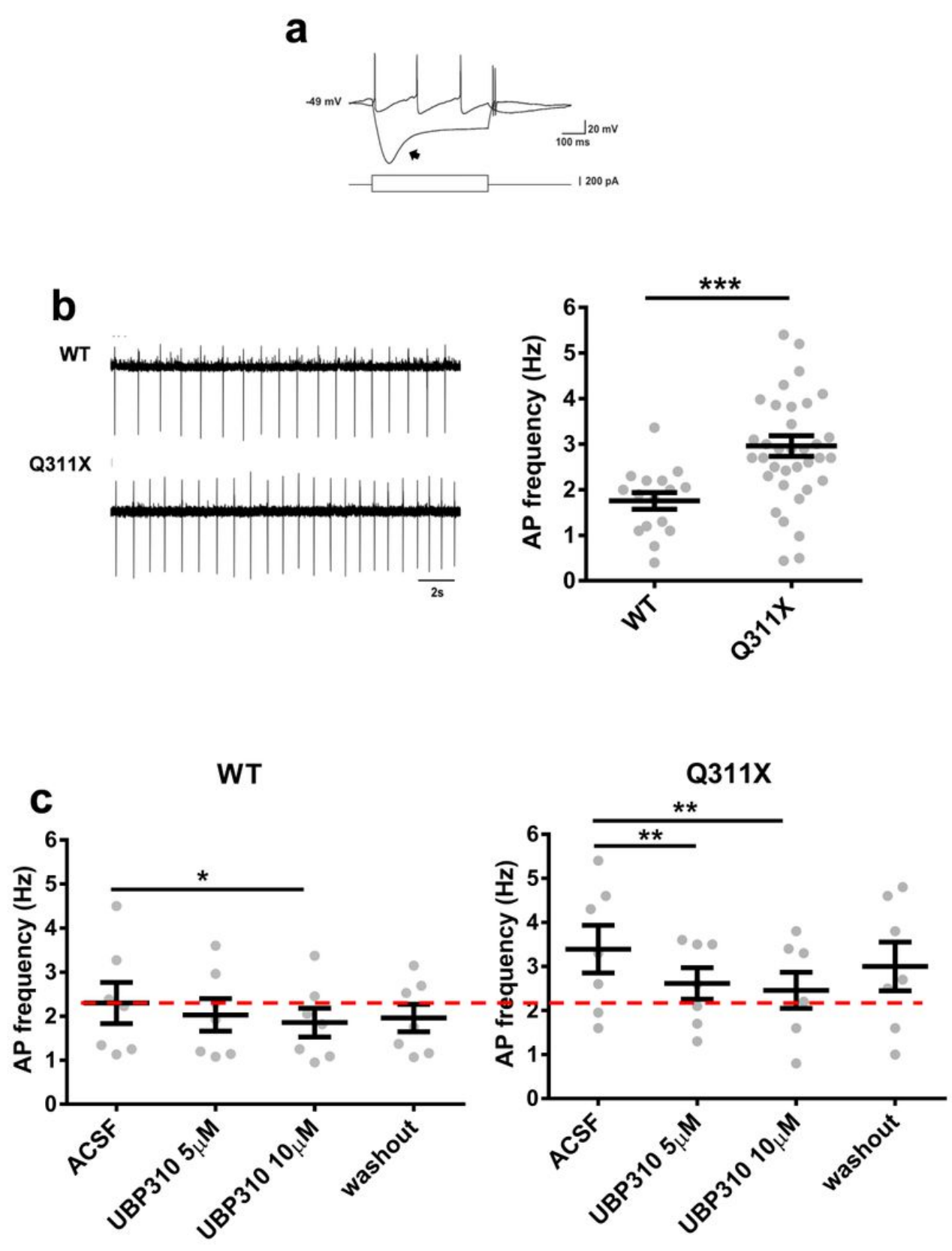

Figure 3

UBP310 normalizes the spontaneous firing activity of SNc DA neurons of parkinQ311X mouse. a) Examples of patch clamp whole-cell recordings in current clamp mode from a SNc DA neuron in acute slice of ventral midbrain. The upper traces show membrane potential hyperpolarizing and depolarizing responses to intracellular injection of negative and positive current steps, respectively ( \pm 200 pA, 500ms; lower traces). Note the relatively depolarized resting membrane potential $(-49 \mathrm{mV})$ and the prominent lh-dependentdepolarizing'sag' (arrow) typical of DA neurons. b) Cell-attached recordings of spontaneous action potential (AP) firing of SNc DA neurons from 
WT and parkinQ311X mice at 25 days of age. The dot plot on the right shows quantification of spontaneous firing frequencies. The mean firing frequency of the SNc DA neurons was significantly higher in parkinQ311X mice compared to WT mice (mean \pm SEM WT: $1.75 \pm 0.18 \mathrm{~Hz}$, parkinQ311X mice: $2.96 \pm 0.23 \mathrm{~Hz}$ ). Data were obtained from 6 WT mice (16 recorded neurons) and 8 parkinQ311X mice (36 recorded neurons). Unpaired Student $t$-test with Welch correction, ${ }^{* \star *} \mathrm{p}=0.0001 \mathrm{t}=4.148 \mathrm{df}=47.75$. c) Summarydot plots showing spontaneous firing frequencies of SNc DA neurons in acute slices of ventral midbrain prepared from WT and parkinQ311X mice at 25 days of age upon extracellular perfusion with UBP310 (5 and 10 MM) and subsequent washout. Mean \pm SEM, WT ACSF 2.3 $\pm 0.46 \mathrm{~Hz}$, WT treated with UBP310 $5 \mu \mathrm{M} 2.03 \pm 0.37 \mathrm{~Hz}$, WT treated with UBP310 10 $\mu \mathrm{M}$ $1.85 \pm 0.32 \mathrm{~Hz}$, WT washout $1.96 \pm 0.31 \mathrm{~Hz} ;{ }^{*} \mathrm{p}=0.02 \mathrm{~F}=7.83$, Friedman repeated measures ANOVA on ranks. Q311X ACSF 3.4 $\pm 0.5 \mathrm{~Hz}$, Q311X treated with UBP310 $5 \mu \mathrm{M} 2.61 \pm 0.36 \mathrm{~Hz}, \mathrm{Q} 311 \mathrm{X}$ treated with UBP310 10 $4 \mathrm{M} 2.46 \pm 0.41$ $\mathrm{Hz}$, Q311X washout 3.0 $\pm 0.55 \mathrm{~Hz}$; one-way repeated measures ANOVA Holm-Sidak test **p=0.009 F=11.87 (7 recorded neurons for each condition). 


\section{Figure 4}

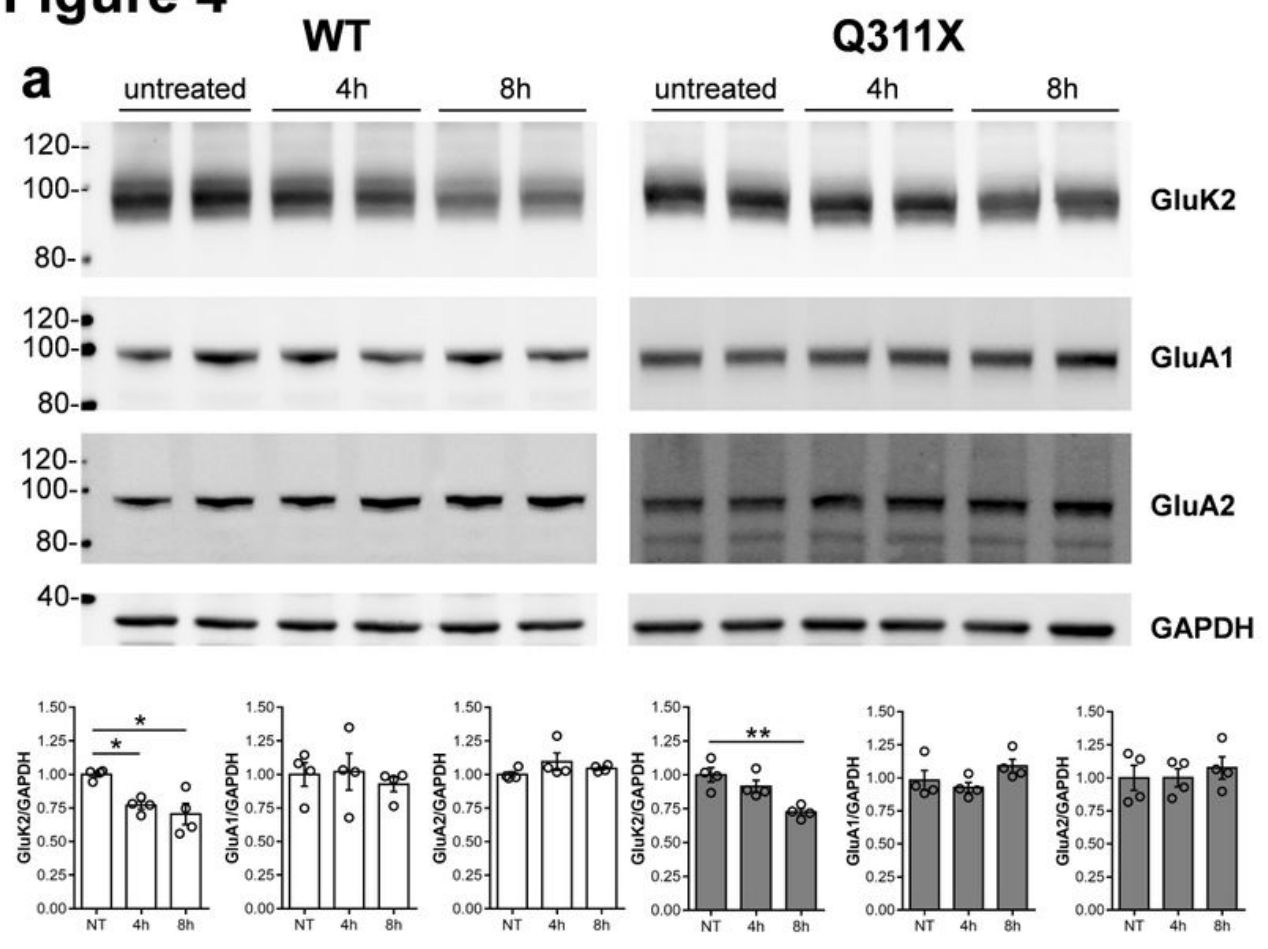

\section{b}
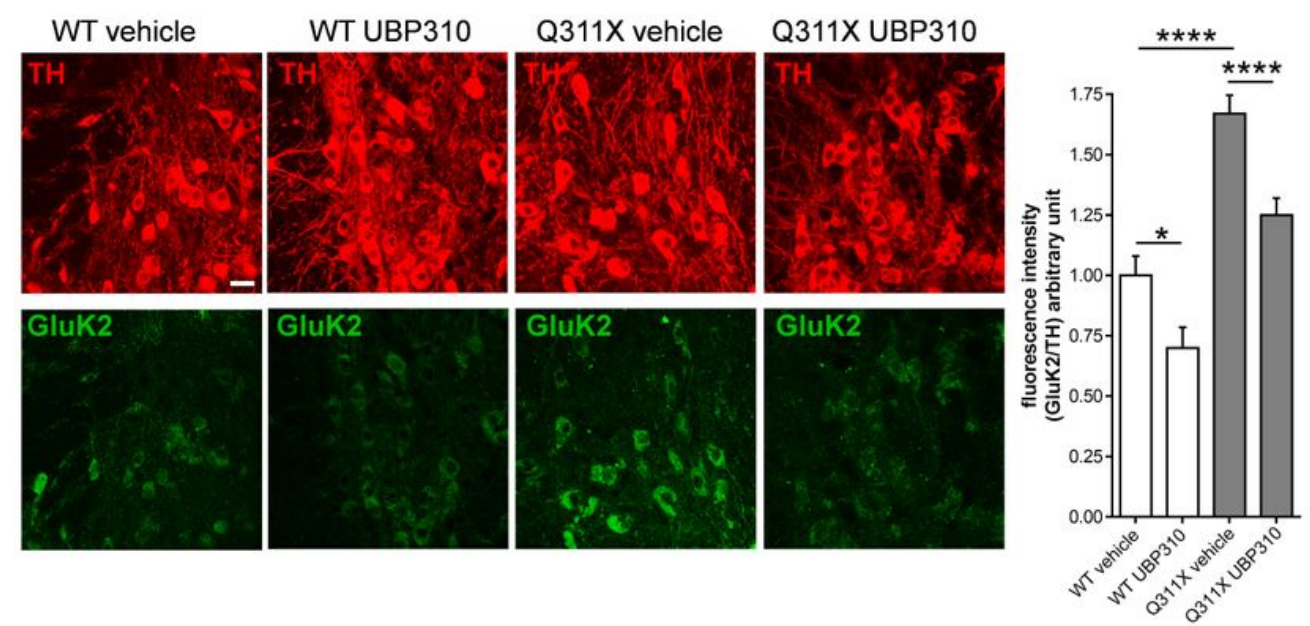

Figure 4

UBP310 decreases the level of GluK2 subunit of KAR. a) Representative Western blot from total brain lysates obtained from WT or parkinQ311X mice treated with $20 \mathrm{mg} / \mathrm{kg}$ UBP310 i.p. for $4 \mathrm{~h}$ or $8 \mathrm{~h}$. The graphs show the means \pm SEM from densitometer quantifications (WT-white bars, Q311X-grey bars). UBP310 treatment induced a decrease in GluK2 levels. WT mice, NT $1.00 \pm 0.02$, $4 \mathrm{~h} 0.77 \pm 0.03$, $8 \mathrm{~h} 0.71 \pm 0.08,4 \mathrm{~h}$ vs NT * $\mathrm{p}=0.047$, 8h vs NT ${ }^{*} \mathrm{p}=0.0011, \mathrm{~F}=7.276$ one-way ANOVA with Bonferroni's test. ParkinQ311X mice, NT $1.00 \pm 0.05$, $4 \mathrm{~h} 0.92 \pm 0.04$, 8h $0.73 \pm 0.02$, 8h vs NT ** $\mathrm{p}=0.0013, \mathrm{~F}=13.36$ one-way ANOVA with Bonferroni's test. UBP310 did not change the levels of AMPA receptor subunits GluA1 and GluA2. b) Representative confocal images showing TH (red) and GluK2 (green) double immunofluorescence in the SNc of WT and parkinQ311X mice treated with vehicle or 
$20 \mathrm{mg} / \mathrm{kg}$ UBP310 i.p. for 8h. The images are representative of 12 sections derived from $\mathrm{n}=3$ WT mice and 12 sections from $n=3$ parkinQ311X mice (GluK2 antibody Millipore 04-921). The bar is 50 $\mu \mathrm{m}$. The histograms show the means $\pm S E M$ from fluorescence intensity quantifications, GluK2/TH: WT vehicle $1.00 \pm 0.08 n=95$ neurons, WT UBP310 0.7 $\pm 0.09 n=91$ neurons,parkinQ311X vehicle 1.67 $\pm 0.08 n=102$, parkinQ311X UBP310 $1.25 \pm 0.07 n=100$, WT vehicle vs. WT UBP310 * $p=0.039, Q 311 X$ vehicle vs. Q311X UBP310 $p=0.0006$,WT vehicle vs. Q311X vehicle $\mathrm{p}=0.00009, \mathrm{~F}=27.95$ one-way ANOVA followed by Tukey's test.

\section{Supplementary Files}

This is a list of supplementary files associated with this preprint. Click to download.

- Figures1.tif

- Figures2.tif

- Figures3.tif

- Figures4.tif

- Figures5.tif 